\title{
AVERAGING A REPRESENTATION OVER A SUBGROUP
}

\author{
R. B. BURCKEL
}

\begin{abstract}
ABSTRACr. The purpose of this note is to extend a well-known technique for getting a representation of a quotient group from one of the original group. This is usually done by "integrating" coefficient functions of the representation over the subgroup, i.e., by applying some mean to them. Hence amenability hypotheses are usually made. None are needed here because the relevant coefficient functions belong to the algebra of weakly almost periodic functions (Eberlein [3]), which is always amenable (Ryll-Nardzewski [5]).
\end{abstract}

This note, whose content is outlined above, was inspired by a recent paper of Ching Chou [2] (whom the author thanks for a preprint). As a corollary to the main result here we are able to deduce his theorem without appeal to several deep results of Eymard [4] which he used; in fact, some of the latter results are also corollaries of the method here.

Let $G$ be a locally compact Hausdorff group, $H$ a closed normal subgroup, $\pi$ : $G \rightarrow G / H$ the projection. Let $U$ be a continuous unitary representation of $G$ on a Hilbert space $\mathscr{H}$ and set $\tilde{\mathcal{C}}=\bigcap_{s \in H} \operatorname{ker}(I-U(s))$. Then

THEOREM. $\tilde{\mathcal{H}}$ is a closed, $U$-invariant subspace of $\mathcal{H}$ and there exists a continuous, unitary representation $\tilde{U}$ of $G / H$ on $\tilde{\mathcal{H}}$ such that

$$
(\tilde{U}(\pi(x)) \tilde{\xi}, \tilde{\eta})=(U(x) \tilde{\xi}, \tilde{\eta}) \quad \forall x \in G, \tilde{\xi}, \tilde{\eta} \in \tilde{\mathcal{H}} \text {. }
$$

Proof. Let $W(H)$ denote the space of weakly almost periodic functions on $H$. There is a two-sided invariant, inversion invariant mean $m$ on $W(H)$. (See [1, p. 15].) Moreover, for each $\xi, \eta \in \mathcal{H}$ the function $h_{\xi, \eta}(t)=(U(t) \xi, \eta), t \in H$ belongs to $W(H)$, being a coefficient function in the continuous unitary representation $U \mid H$ of $H$. (See $\left[1\right.$, p. 36].) Define $B$ on $\mathcal{H} \times \mathcal{H}$ by $B(\xi, \eta)=m\left(h_{\xi, \eta}\right)$. Evidently $h_{\xi, \eta}$ is conjugate bilinear in $(\xi, \eta)$ and so therefore is $B$. Since $\|m\|=1$, we have

$$
|B(\xi, \eta)| \leqslant\left\|h_{\xi, \eta}\right\|_{\infty}<\|\xi\|\|\eta\| \text {. }
$$

Hence there exists a bounded linear operator $P$ on $\mathcal{H}$ such that

$$
B(\xi, \eta)=(\xi, P \eta) \quad \forall \xi, \eta \in \mathcal{H} .
$$

Thus

$$
m_{t}(U(t) \xi, \eta)=(\xi, P \eta) \quad \forall \xi, \eta \in \mathcal{H}
$$

Received by the editors March 19, 1979.

1980 Mathematics Subject Classification. Primary 43A07, 43A30, 43A60, 43A65.

Key words and phrases. Unitary representation, weakly almost periodic function, mean value for WAP functions, Fourier-Stieltjes algebra (nonabelian). 
If $s \in H$ is fixed,

$$
m_{t}(U(t s) \xi, \eta)=m_{t}(U(t) U(s) \xi, \eta) \stackrel{(1)}{=}(U(s) \xi, P \eta)
$$

But also by translation invariance of $m, m_{t}(U(t s) \xi, \eta)=m_{t}(U(t) \xi, \eta)=(\xi, P \eta)$. So

$$
(U(s) \xi, P \eta)=(\xi, P \eta) \quad \forall s \in H, \xi, \eta \in \mathcal{H} \text {. }
$$

Apply the mean $m$ in (2), remembering that $m(1)=1$, and get $m_{s}\left(U(s) \xi, P_{\eta}\right)=$ $(\xi, P \eta)$, then from (1) conclude that $\left(\xi, P^{2} \eta\right)=(\xi, P \eta) \forall \xi, \eta \in \mathcal{C}$. Thus

$$
P^{2}=P \text {. }
$$

Now for any $\xi, \eta \in \mathcal{H}$ we have

$$
\bar{h}_{\eta, \xi}(t)=\overline{(U(t) \eta, \xi)}=(\xi, U(t) \eta)=\left(U\left(t^{-1}\right) \xi, \eta\right)=h_{\xi, \eta}\left(t^{-1}\right)
$$

for all $t \in H$. Since $m$ is inversion invariant and positive (hence real on real functions), we then have

$$
(P \xi, \eta)=\overline{(\eta, P \xi)}=\overline{m\left(h_{\eta, \xi}\right)}=m\left(\bar{h}_{\eta, \xi}\right)=m\left(h_{\xi, \eta}\right)=(\xi, P \eta),
$$

holding for all $\xi, \eta \in \mathscr{H}$. Thus

$$
P=P^{*}
$$

and so $P$ is an orthogonal projection. From (2) we have $\left(\left[I-U\left(s^{-1}\right)\right] \xi, P \eta\right)=0$, whence

$$
(\xi,[I-U(s)] P \eta)=0 \quad \forall \xi, \eta \in \mathcal{K}, s \in H .
$$

Thus $[I-U(s)] P=0 \forall s \in H$,

$$
P(\mathcal{H}) \subset \bigcap_{s \in H} \operatorname{ker}[I-U(s)]
$$

Conversely, if $\eta \in \operatorname{ker}[I-U(s)]$ for every $s \in H$, then

$$
0=\left(\xi,\left[I-U\left(t^{-1}\right)\right] \eta\right)=([I-U(t)] \xi, \eta)=(\xi, \eta)-(U(t) \xi, \eta)
$$

for every $t \in H$, i.e., $h_{\xi, \eta}(t)=(\xi, \eta) \forall t \in H$ and so

$$
(\xi, P \eta)=m_{t}\left(h_{\xi, \eta}(t)\right)=(\xi, \eta) m(1)=(\xi, \eta) \quad \forall \xi \in \mathcal{H},
$$

whence $P \eta=\eta$. From this and (5) we conclude that

$$
P(\mathcal{H})=\bigcap_{s \in H} \operatorname{ker}[I-U(s)]
$$

If $x \in G$ and $s \in H$, then by normality of $H, s^{x}=x^{-1} s x \in H$, so

$$
\begin{aligned}
{[I-U(s)] U(x) } & =U(x)-U(s x)=U(x)-U\left(x s^{x}\right) \\
& =U(x)\left[I-U\left(s^{x}\right)\right] .
\end{aligned}
$$

It follows then from (6) that

$$
P(\mathcal{H}) \text { is invariant under } U(x), \quad \forall x \in G .
$$

From (2) we have for any $x \in G$

$$
(U(s x) \xi, P \eta)=(U(s) U(x) \xi, P \eta) \stackrel{(2)}{=}(U(x) \xi, P \eta) \quad \forall s \in H, \xi, \eta \in \mathcal{H}
$$


and so, setting $\xi=P \zeta$,

$$
\begin{array}{cc}
(P U(s x) P \zeta, \eta)=(P U(x) P \zeta, \eta) & \forall s \in H, x \in G, \zeta, \eta \in \mathcal{K} . \\
P U(s x) P=P U(x) P & \forall s \in H, x \in G .
\end{array}
$$

In particular, $P U(s x) \tilde{\xi}=P U(x) \tilde{\xi} \forall s \in H, x \in G, \tilde{\xi} \in \tilde{\mathcal{H}}$ and so a unitary representation $\tilde{U}$ of $G / H$ is well defined on $\tilde{\mathcal{H}}$ by

$$
\tilde{U}(\pi(x)) \tilde{\xi}=P U(x) \tilde{\xi} \quad \forall x \in G, \tilde{\xi} \in \tilde{\mathscr{H}} \text {. }
$$

Since $\tilde{U} \circ \pi$ is continuous, so is $\tilde{U}$.

COROllaRY (CHOU [2]). With bar denoting closure in the uniform norm and $\tilde{\pi}$ : $C(G / H) \rightarrow C(G)$ being composition with $\pi$, we have

$$
\tilde{\pi}\left(B(G / H)^{-}\right)=\tilde{\pi}(C(G / H)) \cap B(G)^{-} .
$$

Proof. Only " $\supset$ " needs proof. Let $f \in \tilde{\pi}(C(G / H)) \cap B(G)^{-}$and $\varepsilon>0$ be given. Then there exists $h \in B(G)$ such that

$$
|f(x)-h(x)| \leqslant \varepsilon \quad \forall x \in G .
$$

For each $x \in G$ define $h_{x}(t)=h(t x), t \in H$. Since $f \in \tilde{\pi}(C(G / H))$, it is constant on cosets of $H$, so

$$
\begin{aligned}
m_{t}[f(t x)-h(t x)] & =m_{t}\left[f(x)-h_{x}(t)\right]=f(x) m(1)-m\left(h_{x}\right) \\
& =f(x)-m\left(h_{x}\right) .
\end{aligned}
$$

Since $\|m\|=1$, it follows from (10) that

$$
\left|f(x)-m\left(h_{x}\right)\right| \leqslant \varepsilon \quad \forall x \in G .
$$

Now by definition of $B(G), h$ has the form $h(x)=(U(x) \xi, \eta)$ for some continuous, unitary representation $U$ of $G$ on a Hilbert space $\mathcal{H}$ and some $\xi, \eta \in \mathcal{H}$. Then

$$
\begin{aligned}
m\left(h_{x}\right) & =m_{t}(U(t x) \xi, \eta)=m_{t}(U(t) U(x) \xi, \eta) \\
& =(U(x) \xi, P \eta) \quad \text { by }(1) \\
& =\left(U(x) \xi, P^{3} \eta\right) \quad \text { since } P^{2}=P \\
& =\left(P^{2} U(x) \xi, P \eta\right) \quad \text { since } P=P^{*} \\
& =(P U(x) P \xi, P \eta) \quad \text { since } P \text { commutes with } U(x) \\
& =(\tilde{U}(\pi(x)) \tilde{\xi}, \tilde{\eta}) \quad \text { by }(9), \text { with } \tilde{\xi}=P \xi, \tilde{\eta}=P \eta \in \tilde{\mathcal{H}} \\
& =F(\pi(x)), \quad \text { where } F \text { is defined by } \\
& \quad F(H x)=(\tilde{U}(H x) \tilde{\xi}, \tilde{\eta}) .
\end{aligned}
$$

Of course $F \in B(G / H)$, while (11) and (12) say that

$$
|f(x)-\tilde{\pi}(F)(x)|<\varepsilon \quad \forall x \in G .
$$

Since $\varepsilon>0$ is arbitrary, this shows that $f \in \tilde{\pi}(B(G / H))^{-}$.

SCHOLIUM (EyMARD [4]). $\tilde{\pi}(B(G / H))=\tilde{\pi}(C(G / H)) \cap B(G)$. 
Proof. Only " $\supset$ " needs proof. Let $f \in \tilde{\pi}(C(G / H)) \cap B(G)$ be given. Then for the $h$ in the last proof we can take $f$ itself. Thus $h$ is constant on cosets of $H$ and

$$
\begin{aligned}
m\left(h_{x}\right) & =m_{t}(U(t x) \xi, \eta)=m_{t}((U(x) \xi, \eta) \cdot 1) \\
& =(U(x) \xi, \eta)=h(x) \quad \forall x \in G .
\end{aligned}
$$

It follows from (12) that

$$
f(x)=h(x)=F(\pi(x))=\tilde{\pi}(F)(x) \quad \forall x \in G,
$$

so $f=\tilde{\pi}(F) \in \tilde{\pi}(B(G / H))$.

\section{RefERENCES}

1. R. B. Burckel, Weakly almost periodic functions on semigroups, Gordon and Breach, New York, 1970.

2. C. Chou, Uniform closures of Fourier-Stieltjes algebras, Proc. Amer. Math. Soc. 77 (1979), 99-102.

3. W. F. Eberlein, Abstract ergodic theorems and weak almost periodic functions, Trans. Amer. Math. Soc. 67 (1949), 217-240.

4. P. Eymard, L'algèbre de Fourier d'un groupe localement compact, Bull. Soc. Math. France 92 (1964), 181-236.

5. C. Ryll-Nardzewski, On fixed points of semigroups of endomorphisms of linear spaces, Proc. of the Fifth Berkeley Sympos. on Mathematical Statistics and Probability (1965/66), vol. II, Part I: Theory of Probability, Univ. of California Press, Berkeley, 1967.

Department of Mathematics, Kansas State University, Manhattan, Kansas 66506 